\title{
„Whistleblowing“ eines Angestellten Schadensersatz für eine Klinik nach Umsatzrückgang?
}

Die Schadensersatzklage eines Krankenhauses gegen eine dort beschäftigte Ärztin und deren Lebensgefährten wegen anonym erstatteter Strafanzeigen, deshalb durchgeführter staatsanwaltschaftlicher Durchsuchung und anschließender Pressekampagne und damit verbundenem Umsatzrückgang wurde vom Landesarbeitsgericht Hamm mit Urteil vom 21.07.2011 (Az.: 11 Sa 2248/10) abgewiesen. Die Revision beim Bundesarbeitsgericht gegen dieses Urteil läuft.

Nicht selten fühlen Angestellte, die ihr Arbeitsverhältnis mit dem Krankenhaus nicht einvernehmlich beendet haben, sich „berufen“, über Missstände, die ihnen während ihrer Tätigkeit aufgefallen sind, nachträglich zu berichten.

Im Englischen gibt es für dieses Verhalten der Offenlegung von Missständen in Unternehmen einen stehenden Begriff, das sogenannte „whistleblowing“. Mit seinem Urteil betritt das Landesarbeitsgericht (LAG) Hamm juristisches Neuland, indem es sich erstmals zu einer etwaigen Schadensersatzpflicht eines Anzeigeerstatters rechtlich äußert.

\section{Der Fall}

Klägerin ist ein Universitätsklinikum, das eine ehemalige Chefärztin und deren Lebensgefährten auf Schadensersatz wegen rückläufiger Patientenzahlen in ihrer Klinik für Thorax-, Herz- und Gefäßchirurgie (THG) ab dem Sommer 2008 verklagt. Die beklagte Chefärztin war Herzchirurgin und stand vom 01.07.2007 bis 30.09.2008 in einem Arbeitsverhältnis zu der Universitätsklinik. Der zweite Beklagte war der Lebensgefährte der Chefärztin, der nicht über eine ärztliche Ausbildung verfügt. Geplant war, dass die Chefärztin spätestens ab 2011 die Nachfolge des dann in Ruhestand gehenden Klinikdirektors Prof. S. antreten sollte. Seit Anfang Oktober 2007 kam es bereits zu Auseinandersetzungen zwischen Prof. S. und weiteren ärztlichen Mitarbeitern der THG und der beklagten Chefärztin. Daraufhin kündigte das Universitätsklinikum das Arbeitsverhältnis mit der Chefärztin mit Wirkung zum 31.12.2007. Gegen diese Kündigung erhob die Chefärztin Kündigungsschutzklage vor dem Arbeitsgericht. Im März 2008 schloss das Universitätsklinikum mit der Chefärztin einen Vergleich.
Bereits Ende Dezember 2007 legte die Chefärztin im Rahmen eines Gesprächs mit der Rektorin der Universität eine von ihr erstellte Liste vor, die im Wesentlichen die Wiedergabe der Operationsverläufe bei 13 Patienten beinhaltete und daneben weitere Kritikpunkte thematisierte. Angesprochen wurden z.B. Punkte wie „Kein DRG-gerechtes Entlassungsmanagement (s. Brief W1: 100\%ige Belegung erwünscht)”, „Rassismus, Menschenunwürdigkeit”, „Jungoperateure“ etc. Mit dieser Liste wollte die Chefärztin Qualitätsmängel in der THG belegen.

Im April 2008 erhielt der damalige zuständige Minister des Landes NRW über den Vater eines weiteren Arztes aus der Abteilung ein Schreiben, in dem er mitteilte, er verfüge über Informationen über erhöhte Anzahlen von Komplikationen bis hin zu Todesfällen in der THG, die von Prof. S. zu vertreten seien. Der Minister wurde aufgefordert, sich persönlich um die Problematik zu kümmern, ansonsten werde der Unterzeichner des Briefes „keinen Aufwand scheuen, meine ausgedehnten Verbindungen zu Politik und vor allem zu Presse und Medien zu nutzen, um diese Zustände in der Herzchirurgie im UKM (...) an die Öffentlichkeit zu bringen, sollte es nicht in allerkürzester Zeit adäquate „innere” Lösungen der Problematik geben". Vom Ministerium wurde eine Kommission eingesetzt, die die Vorwürfe nicht bestätigen konnte.

Ab Juni 2008 wurden wiederholt anonyme Strafanzeigen zu Vorfällen in der THG erstattet. Am 17.06.2008 ging bei der Generalstaatsanwaltschaft Hamm eine anonyme Strafanzeige gegen Prof. S. wegen vorsätzlicher Körperverletzung mit Todesfolge bezogen auf eine Patientin ein. Es folgten diverse weitere anonyme Strafanzeigen. Neben den Strafanzeigen gab es anonyme Schreiben an Angehörige, Pa- tienten, den Südwestrundfunk SWR und den Vater eines Arztes der THG. Die Staatsanwaltschaft leitete ein Ermittlungsverfahren ein. Am 17.07.2008 fand eine polizeiliche und staatsanwaltschaftliche Durchsuchung in den Diensträumen der Klinik und in der Privatwohnung von Prof. S. statt. Die Staatsanwaltschaft unterrichtete nach dieser Aktion die Medien über die am selben Tag erfolgten Durchsuchungshandlungen. Dies führte zu einer von der Klägerin als „verheerend” bezeichneten Berichterstattung in den Medien und zahlreichen Presseartikeln. Das gegen Prof. S. eingeleitete Ermittlungsverfahren wurde von der Staatsanwaltschaft im November 2008 wegen erwiesener Unschuld eingestellt. Im Rahmen der Ermittlungen entstand der Verdacht, dass die Chefärztin und ihr Lebensgefährte Urheber der anonymen Schreiben seien. Im September 2008 fand eine Durchsuchung in den Diensträumen der Chefärztin, die mittlerweile anderweitig tätig war, und ihrer Privatwohnung statt. Der Lebensgefährte gestand in einem Schreiben vom September 2008 ein, Verfasser der anonymen Schreiben zu sein. Ende September legte er hierzu ein Geständnis ab. Er erklärte hierbei, die Chefärztin sei an den Schreiben in keiner Weise beteiligt gewesen.

Im Januar 2009 erhob die Staatsanwaltschaft gegen beide Anklage wegen Verstoßes gegen das Bundes- und das Landesdatenschutzgesetz, Verletzung von Privatgeheimnissen, falscher Verdächtigung, Verleumdung sowie versuchter Nötigung. Die Anklage wurde zugelassen, die Hauptverhandlung jedoch wegen vordringlicherer Strafsachen zunächst verschoben.

Mit Schreiben vom Juli 2009 machte die Universitätsklinik gegenüber der Chefärztin einen Schadensersatzanspruch in Höhe von über 3 Millionen Euro geltend. Dieser Anspruch wurde von ihr zurückgewiesen. Im Februar 2010 verklagte das Krankenhaus die Ärztin und deren Lebensgefährten zunächst auf einen Teilbetrag der Schadenssumme in Höhe von 1,5 Millionen Euro. Die Schadensersatzforderungen werden darauf begründet, dass die Berichterstattungen, die durch die anonymen Anzeigen und Schreiben hervorgerufen worden seien, für das Klinikum verheerende Folgen gehabt hätten. Die Patientenzahlen seien dramatisch zurückgegangen, diverse Chefärzte und nachgeordnete Oberärzte, die Patienten 
der THG zuwiesen, hätten angerufen und mitgeteilt, dass Patienten sich wegen der aktuellen Berichterstattung geweigert hätten, sich in die THG einweisen zu lassen bzw. dass auf Verlangen von Patienten bereits vereinbarte Behandlungen hätten abgesagt werden müssen. Insbesondere die Zuweisungen durch ein bestimmtes Krankenhaus seien im Jahr 2008 im Vergleich zum Jahr 2007 um ca. $40 \%$ zurückgegangen. Der dortige Chefarzt habe Patienten wesentlich zeitaufwendiger beraten müssen, um das Misstrauen bei den Patienten gegenüber der THG auszuräumen. Auch ein auswärtiger Einweiser habe aufgrund der medialen Berichterstattung ab Juli 2008 in erheblichem Umfang von Einweisungen absehen müssen, da sich die Patienten geweigert hätten, sich in die THG einweisen zu lassen.

Die Chefärztin verteidigte sich in dem Verfahren dahingehend, dass sie von den Vorgängen nichts gewusst habe. Sie habe die behaupteten Schreiben nicht verfasst. Außerdem sei durch den Lebensgefährten nicht in der Absicht gehandelt worden, der Universitätsklinik einen Schaden zuzufügen. Das Arbeitsgericht wies die Klage in erster Instanz ab. Hiergegen wurde von dem Universitätsklinikum Berufung eingelegt. Die Chefärztin und ihr Lebensgefährte beantragten im Rahmen einer Anschlussberufung die Feststellung, dass sie über den mit der Teilklage geltend gemachten Betrag hinaus keinen weiteren Schadensersatz zu leisten hätten.

Das LAG Hamm entschied über die Berufung wie folgt:

Die Berufung des Universitätsklinikums hat keinen Erfolg, die Chefärztin und ihr Lebensgefährte schulden der Universitätsklinik keinen Schadensersatz in der eingeklagten Höhe. Damit wurde der Anschlussberufung der Ärztin und ihres Lebensgefährten automatisch Recht gegeben.

Gegen dieses Urteil wurde Revision zum Bundesarbeitsgericht zugelassen.

\section{Die Entscheidung}

Das Landesarbeitsgericht Hamm entschied, dass dem Universitätsklinikum kein Schadensersatz gegen die Chefärztin und ihren Lebensgefährten zustehe, da kein pflichtwidriges Verhalten vorgelegen habe.

Der Forderung des Universitätsklinikums gegen den Lebensgefährten stehe entgegen, dass die vom Klinikum zur Grundla- ge ihrer Ersatzforderung genommene Presseberichterstattung auf die Berichterstattung der Staatsanwaltschaft über das von dort geführte Ermittlungsverfahren zurückgeht. Das Ermittlungsverfahren sei zwar „zumindest auch“ durch die von dem Lebensgefährten anonym erstatteten Strafanzeigen ausgelöst worden. Für Schäden, die durch die Erstattung einer Strafanzeige und ein daraus resultierendes staatliches Ermittlungsverfahren verursacht sind, hätte der Anzeigeerstatter jedoch nur unter besonderen Voraussetzungen Ersatz zu leisten.

Diese Voraussetzungen seien hier nicht erfüllt.

\section{Kein rechtswidriges und schuld- haftes Verhalten}

Da der Lebensgefährte anders als die Chefärztin zu keinem Zeitpunkt in einem Arbeitsverhältnis zur Klinik gestanden habe, können Schadensersatzansprüche gegen ihn nicht auf vertraglicher, sondern nur auf gesetzlicher Grundlage bestehen. Den in Frage kommenden Anspruchsgrundlagen sei gemeinsam, dass der Schaden, dessen Ersatz gefordert wird, durch ein rechtswidriges und schuldhaftes Verhalten des Anspruchsgegners verursacht sein muss. Zwischen dem rechtswidrigen und schuldhaften Verhalten des Schädigers und der Rechtsbeeinträchtigung muss ein Ursachenzusammenhang bestehen. Die vom Klinikum zugrunde gelegte Presseberichterstattung, die für den Schaden verantwortlich gemacht wird, geht zurück auf die Berichterstattung der Staatsanwaltschaft. Dass daneben die anonymen Schreiben des Lebensgefährten an Patienten, Angehörige von Patienten und Angehörige von Ärzten unabhängig von der Pressearbeit der Staatsanwaltschaft zu einer Berichterstattung in den Medien geführt hätten, sei nach dem unterbreiteten Sachverhalt nicht festzustellen. Außerdem ergebe sich nicht, dass Schreiben des Lebensgefährten unmittelbar an Medien zu einer Presseveröffentlichung geführt hätten. Das umfangreiche anonyme Anschreiben an den Südwestrundfunk habe unstreitig nicht zu einer Berichterstattung geführt. Wegen des anerkennenswerten Interesses der Allgemeinheit an der Aufklärung von Straftaten begründet die Erstattung einer Anzeige bei der Polizei oder bei der Staatsanwaltschaft nur unter besonderen Voraussetzungen eine Schadensersatzverpflichtung des Anzeigeerstatters.

\section{Anzeige darf nicht wissentlich unwahr oder leichtfertig sein}

Das Gericht bezieht sich in seiner Begründung im Wesentlichen auf Grundsätze die im Zusammenhang mit Kündigungen bezüglich Strafanzeigen von Arbeitnehmern gegen ihren Arbeitgeber standen. Für die Frage, ob die Erstattung der Strafanzeige durch einen Arbeitnehmer einen Kündigungsgrund für den Arbeitgeber bilden kann, käme es nach der Rechtsprechung des Bundesarbeitsgerichts nicht entscheidend darauf an, ob die Anzeige zu einer Verurteilung geführt hat oder nicht. Es sei gerade Sinn der Einleitung eines Ermittlungsverfahrens, die bei Anzeigeerstattung notwendigerweise offene Frage der Tatbegehung erst zu klären. Eine Strafanzeige sei vor diesem Hintergrund in der Regel nur dann nicht mehr als berechtigt anzusehen, wenn der Arbeitnehmer schon bei der Erstattung der Anzeige weiß, dass der erhobene Vorwurf nicht zutrifft oder dies jedenfalls nicht leicht erkennen kann oder einen unverhältnismäßigen Gebrauch von seinem Recht macht. Ähnliche Grundsätze hätte der Europäische Gerichtshof für Menschenrechte aufgestellt. Das LAG Hamm verweist auch noch auf eine Bundesverfassungsgerichtsentscheidung, die sich mit einer schadensersatzrechtlichen Fragestellung bei gutgläubigen Strafanzeigeerstattern auseinandersetzt. Nach Auffassung des Bundesverfassungsgerichts müsse beachtet werden, dass mit der Erstattung einer Strafanzeige eine von der Rechtsordnung erlaubte und gebilligte Möglichkeit wahrgenommen wird. Die Strafanzeige eines Bürgers liege im allgemeinen Interesse an der Erhaltung des Rechtsfriedens und an der Aufklärung von Straftaten, es sei denn, die Anzeige wird wissentlich unwahr oder leichtfertig ohne erkennbaren Grund erstattet. Mit den Grundgeboten des Rechtsstaats sei es nicht vereinbar, wenn derjenige, der in gutem Glauben und nicht leichtfertig ohne erkennbaren Grund eine Strafanzeige erstattet hat, Nachteile dadurch erleidet, dass sich seine Behauptung nach behördlicher Prüfung als unrichtig oder nicht aufklärbar erweist. Die nicht wissentlich unwahr oder leichtfertig ohne erkennbaren Grund erstattete Strafanzeige erfolge in einem rechtsförmlichen Verfahren und sei deshalb nicht rechtswidrig und begründe keine Schadensersatzverpflichtung. Nach Auffassung der Berufungsrichter konnte im konkreten Fall nicht festgestellt werden, dass der Lebensgefährte die Straf- 
anzeigen wissentlich unwahr oder leichtfertig ohne erkennbaren Grund erstattet hätte. Hierfür habe das Universitätsklinikum auch keine Tatsachen vorgetragen. Da nicht festgestellt werden könne, dass die Strafanzeigen bewusst wahrheitswidrig oder leichtfertig erstattet worden seien, sei der Lebensgefährte nicht zum Schadensersatz verpflichtet. Daran ändere auch der Umstand nichts, dass mit den zahlreichen anonymen Schreiben an andere Adressaten, mit der polemischen Wortwahl und den teils drastischen Verunglimpfungen sowohl in jenen Schreiben als auch in den Schreiben an die Staatsanwaltschaft belegt sei, dass es dem Autor der Schreiben nicht allein um den Schutz von Allgemeininteressen ging, sondern, dass das Ziel verfolgt worden sei, die THG und namentlich genannte Ärzte der THG bei Patienten, Angehörigen, Medien und Strafverfolgungsbehörden in ein schlechtes Licht zu stellen. Da die Voraussetzungen eines Schadensersatzanspruches gegen den Lebensgefährten nicht gegeben seien, könne dahingestellt bleiben, ob die Presseberichterstattung im Sommer 2008 tatsächlich für wirtschaftliche Einbußen bei der THG ursächlich geworden seien, ob eine Einbuße in der von der Klägerin behaupteten Höhe eingetreten sei und ob die Klägerin zureichenden Tatsachenvortrag zur Schadensfestlegung gehalten habe.

\section{Auch Ärztin handelte in gutem Glauben}

Auch der Schadensersatzanspruch gegen die Chefärztin wurde vom LAG Hamm zurückgewiesen. Auch bei ihr könne nach dem unterbreiteten Tatsachenstoff nicht festgestellt werden, dass die anonymen Anzeigen an die Staatsanwaltschaften wider besseres Wissen oder leichtfertig erstattet worden seien. Dies gelte auch dann, wenn berücksichtigt werde, dass die Ärztin, anders als ihr Lebensgefährte, über profunden ärztlichen Sachverstand verfügte.

Wegen der grundsätzlichen Bedeutung der entscheidungserheblichen Frage der Schadensersatzpflicht in Fällen des „whistlebowling“ wurde die Revision zum Bundesarbeitsgericht zugelassen. Das Verfahren wird beim Bundesarbeitsgericht unter dem Az.: 8 AZR 88/11 geführt. Der vorgesehene Verhandlungstermin ist der 13.12.2012.

\section{Fazit}

Das LAG Hamm beschäftigt sich erstmals mit der Frage einer möglichen Schadensersatzpflicht eines Hinweisgebers von Missständen. Auch wenn eine derartige Schadensersatzpflicht vom Gericht verneint wird, muss festgehalten werden, dass das Urteil noch nicht rechtskräftig ist. Zu berücksichtigen ist bereits jetzt, dass bewusst wahrheitswidrig oder leichtfertig erstattete Anzeigen durchaus zu einer Schadensersatzpflicht führen können. Jeder Anzeigeerstatter läuft also Gefahr, bei völlig ungerechtfertigten Anzeigen tatsächlich in Anspruch genommen zu werden. Das Urteil ist also kein Freibrief, einen unliebsamen Arbeitgeber „hinzuhängen“. Im Übrigen ist es sicherlich ratsam, abzuwarten, ob die oberstgerichtliche Rechtsprechung das Urteil bestätigt. Unklar ist im Übrigen der Ausgang der gegen die Chefärztin und ihren Lebensgefährten eingeleiteten Strafverfahren!

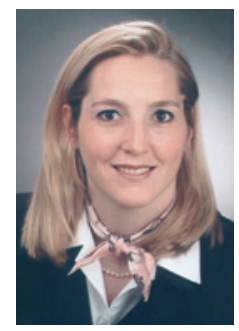

Korrespondenz

Dr. iur. Isabel Häser Rechtsanwältin

Ehlers, Ehlers und Partner

Widenmayerstr. 29

80538 München 